\title{
English Grammar Discrimination Training Network Model and Search Filtering
}

\author{
Juan Zhao \\ School of Foreign Studies, North China University of Water Resources and Electric Power, Zhengzhou, Henan 450046, China \\ Correspondence should be addressed to Juan Zhao; juanzhao@ncwu.edu.cn
}

Received 6 January 2021; Revised 19 April 2021; Accepted 26 April 2021; Published 4 May 2021

Academic Editor: Wei Wang

Copyright ( 92021 Juan Zhao. This is an open access article distributed under the Creative Commons Attribution License, which permits unrestricted use, distribution, and reproduction in any medium, provided the original work is properly cited.

\begin{abstract}
The statistics-based method ignores the semantic constraints in the English grammar area branch training model and is unable to identify the orientation information effectively. This paper systematically discusses the close relationship between English grammar area branch training model filtering, English grammar area branch training model retrieval, and machine learning. By analyzing the role of the situation in the understanding of the English grammar area branch training model, the relationship between the English grammar area branch training model and situation model and the correlation between the features of the English grammar area branch training model and situation model are determined, and then, a set of filtering methods for the English grammar area branch training model are proposed. At present, there are few research studies on bias filtering, and the method of thematic filtering is generally used, which has poor effect. This paper makes full use of the domain knowledge and adopts the semantic pattern analysis technology to establish a wealth of semantic analysis resources, including various dictionaries, rules, and weight representation, so as to effectively filter the inclined English grammar area branch training model. The introduction of semantic data sources solves the problem of data sparsity and cold start in the traditional collaborative filtering system. In addition, in order to improve the scalability and real-time performance of the recommendation system, the data mining method is used to perform fuzzy clustering for users and projects in the offline data preprocessing stage. This paper proposes a search and filter scheme based on the orientation of the training model in English grammar area, elaborates on the details, constructs a whole set of function structure from representation to weight, and gives the experimental results, which prove that the system has a good filtering effect and is fast. Compared with the traditional statistical methods, the results are satisfactory.
\end{abstract}

\section{Introduction}

English grammar is the law of language, reflecting the structure and organization of language. English grammar is not only the language knowledge and the ability to accurately use the grammatical structure but also a skill requiring production training, involving form, meaning, and use. English grammar can promote the understanding and monitor the output of language learning, and the cultivation of grammar awareness can help learners pay attention to the characteristics of language input. In the appropriate learning stage [1], providing learners with grammar knowledge in an appropriate form is conducive to improving their language learning effect. Without the knowledge of English grammar, meaningful communication cannot be realized. Neglecting grammar teaching will affect the improvement of learners' language ability.

The search filtering algorithm based on the Network model of English grammar discrimination training scores the nearest user or item prediction, so the efficiency of online recommendation is affected by the number of users or items. This paper introduces the random forest model that can be trained offline, proposes to construct the training dataset of the supervised learning model by using the scoring matrix and the user and project membership matrix obtained previously through clustering, trains the random forest model, and uses the offline training model to predict the scoring when online recommendation is made. Based on the defects of search filtering based on statistics, combined with the search filtering technology based on text orientation 
analysis, as well as related schemes and work, a whole set of function structure from representation to weight is constructed and verified by experiments.

\section{Related Work}

Classroom teaching should pay enough attention to and give due status to English grammar. However, under the principles of teaching language instead of teaching language knowledge and fluency over correctness, college English grammar teaching and learning are faced with many difficulties. These difficulties are mainly manifested as follows: teachers seldom involve grammar in class, dilute grammar, and even reject grammar teaching because they believe that students have learned grammar in middle school and it is unnecessary for them to teach grammar in college. The students have vague grammar knowledge and rules and cannot skillfully use grammar. They seem to be fluent in spoken and written language, but in fact, they make many grammatical mistakes. They are not aware of their mistakes in speaking or writing. Quite a few students do not realize the important role of grammar knowledge and grammar application ability in the second language learning and seldom attribute their insufficient or poor performance of language knowledge and skills to the defects of grammar knowledge and grammar application ability [2]. In recent years, the problem of grammar teaching and learning are causing the predecessors and scholars focus in foreign language and thought: a fluent language seems to make many learners wrong, and it is difficult to move in the right direction by his own learning because he has made a mistake, and he still thinks his bad habits are correct, and he behaves decently with some wrong expressions $[3,4]$. To really master English, we must master the basic knowledge of grammar. If you do not know grammar, you cannot speak English. The purpose of learning grammar is to use the language more effectively in communication. The more complex the communication task, the higher the requirement for language accuracy and the greater the dependence on grammar $[5,6]$.

Unless students have a solid foundation in middleschool English grammar to enable them to carry out language learning at a higher level, grammar teaching must be carried out in college [7]. However, there are few empirical studies on the teaching and learning of English grammar. Foreign language major core journals in China on the knowledge of English grammar teaching and learning found that although some research, respectively, discusses the English grammar knowledge to the predictive power of pragmatic knowledge $[8,9]$ ability to distinguish between training and development trend, English grammar [10], college English teachers and students grammar teaching and learning belief [11], and English grammar knowledge and English and English language skills such as the potential relationship[12], due to adopting the concept of English grammar knowledge or ability and measuring tools, testing the level of task difficulty and subjects of English language levels vary. The result is not the same. So far, it is rare to find a scientific, standardized, and easy-to-use test tool for
English grammar discrimination, which is suitable for English majors in China. Because of this, trying to prepare the ability to distinguish between English learners' English grammar training scale (hereinafter referred to as the English grammar to distinguish ability training scale) $[13,14]$ was to understand English major junior learners' English grammar knowledge and skill development and test the ability of using their knowledge of English grammar and syntax and strengths and weaknesses for English professional basic course teachers and learners of English to take timely remedial strategy to provide targeted intervention measurement tools. It also provides a research tool for Chinese foreign language education researchers to explore the development trend, performance characteristics, individual differences, and the potential relationship between college English learners' English grammar discrimination ability training and English language skills. Researchers have focused their attention on the study of the understanding of the automatic filtering system, and the development of corpus linguistics has also provided new opportunities and challenges for the study of the understanding of the filtering system $[15,16]$.

However, due to the length, understanding technology is the key to understanding information processing, which is also the core problem of natural language processing. Relevant technologies for length understanding are not yet mature, and a series of problems, such as what means to be used for grammar and semantic analysis and what model has to be used to represent length content, have not been completely solved [17]. The existing methods of understanding information processing are generally superficial understanding, and it is inevitable that the text field dealt with will be limited [18]. English grammar search filtering technology has gained widespread attention in the industry in recent years. This is mainly due to the rapid development of the Internet and the increasing demand for Chinese information on the Internet at an unprecedented rate and the rapid increase in the number of Chinese Internet users. All of these put forward higher and newer requirements for English grammar search filtering $[19,20]$. As far as the Chinese-oriented search filter system is concerned, its development is still in the preliminary stage. Whether various filtering algorithms oriented to English are suitable for English grammar search and filtering remains to be tested by experiments. Some need to be improved, while others may need to be developed in a different way [21-23]. Although researchers have also proposed some important methods and ideas, such as the information streaming mechanism [24] and exemplar-based English grammar search filtering model [25-27], the experimental system developed has achieved different filtering accuracies in different fields.

\section{Network Pattern Design of English Grammar Discriminative Training}

3.1. Framework of the English Grammar Discriminant Training Model. The ability to distinguish English grammar is the ability to understand, monitor, operate, and produce English sentences at the sentence level. Grammatical 
competence is composed of different grammatical skills, including sentence monitoring skills, sentence operation skills, and sentence generation skills, each of which is based on different grammatical subskills, as shown in Figure 1.

Among the grammatical skills, the sentence monitoring technology is proficient in using English grammar knowledge and grammar rules to identify and correct the incorrect use of English lexical knowledge and syntactic knowledge in the target sentence, including lexical monitoring and syntactic monitoring subskills. Sentence operation skill refers to the ability to combine several simple sentences with compound sentences and convert clauses into phrases, phrases into clauses, and declarative sentences into special sentence patterns by using English grammar knowledge and rules. It includes sentence integration subskill and sentence transformation subskill. Sentence generation skill refers to the ability to skillfully use English grammar knowledge and grammar rules to produce semantic and logical corresponding sentences in the target language (English) according to the sentence content of the source language (English grammar discrimination training), which is mainly manifested as the ability to translate Chinese sentences into English.

The design framework of English grammar discriminating ability training scale is based on the operational definition of English grammar discriminating ability training. Scale for measuring target involves the grammar and syntax used, measuring project monitoring skill of sentences, testing contents including lexical monitoring and skills and syntactic monitoring and skills, integrating the skills, and shifting the sentence translation skills and words and skills, and the test type contains the error identification of a merger with correction, sentences, sentence pattern conversion, and the induction of translation. The overall description of the scale design framework is shown in Table 1.

The bidirectional breakdown of English Grammar ability scale is drawn up according to the design framework of the scale. The scale consists of 90 questions, including 41 for sentence monitoring skills measurement, 35 for sentence operation skills measurement, and 15 for sentence generation skills measurement. Each question is worth between 1 and 5 points for a total of 135 . Rating scale can be a complete measuring, testing 100 minutes total time limit (including test instructions, reading, and answering the questions on demonstration time), or it can be divided into five times to complete, followed by sentence monitoring skills, integrating the skills, shifting the skills, and sentence skills test, the time limit of 20 minutes (including test instructions, reading, and answering the questions on demonstration time). The detailed bidirectional breakdown table is shown as Table 2.

English grammar to distinguish between the training comprehensive analysis method is mainly aimed at the analysis of the traditional basic method is put forward with the respective defects of sentence, including the analysis of the traditional basic method including the component analysis of the sentence and sentence analytic hierarchy process, and gradually developed to combine the two analysis methods, complementing each other, forming a comprehensive analysis. Because of its guiding significance to the syntactic analysis of English grammar discrimination training, the thought of synthetic analysis can be introduced into dependency syntactic analysis.

\subsection{Design of the English Grammar Discriminant Training} Network Model. The structural types of English grammar discrimination training reflect the general law of syntactic combination among the components of English grammar discrimination training. The English grammar structure template designed in this paper is a series of related conversion rules composed of the English grammar structure differentiation training and differentiation. It will serve as a bridge (product) for the combination of comprehensive analysis thought and syntactic analysis. The preprocessing results of the LTP sentence dependency parsing method based on the neural network model can be converted into the main analysis results which conform to the principle of dependency semantics and dependency syntax. Therefore, the design of the template for distinguishing training structure types in English grammar plays an important role in obtaining the results of syntactic dependency analysis with both semantic and structural considerations. The training network model architecture diagram is shown in Figure 2.

Taking the structure types of English grammar to distinguish training templates as the idea, the comprehensive analysis combined with the bridge of dependency syntax analysis may result in the interdependence analysis of both structure and semantics among the components of clauses, but how to realize the computer input sentences according to the matching conditions for English grammar to distinguish the training structure type template rule automatic conversion becomes very important. In fact, the automatic conversion process of the input sentences to distinguish the structure type template rules according to the matching conditions is a subgraph isomorphism problem related to the attribute relation graph. Therefore, in view of the obvious advantages of VF2 algorithm in dealing with the isomorphism problem of subgraphs on the attribute relation graph, this section introduces the idea of VF2 algorithm to realize the comprehensive analysis and postprocessing of the preprocessing results of dependency analysis based on the neural network model and finally obtains the dependency analysis results of each component in the clause with both structure and semantics.

VF2 algorithm is used in the network model of English grammar discriminative training. The search process is represented by the state, and the pruning strategy is added in the search process. Generally, in the matching process of pruning the state description of the search tree using a heuristic search strategy, it is assumed that the target graph G1 and the query subgraph G2 use the state s to store partial matches during the search process. $\mathrm{M}$ (s between any states) represents a part of the matching set, and the contained $M(s)$ is mapped in the form of (n, M) to determine whether to join the state. The new mapping of $s$ (n. M) mainly depends on the feasibility of the return value of the function $F(s, n, M)$; if 


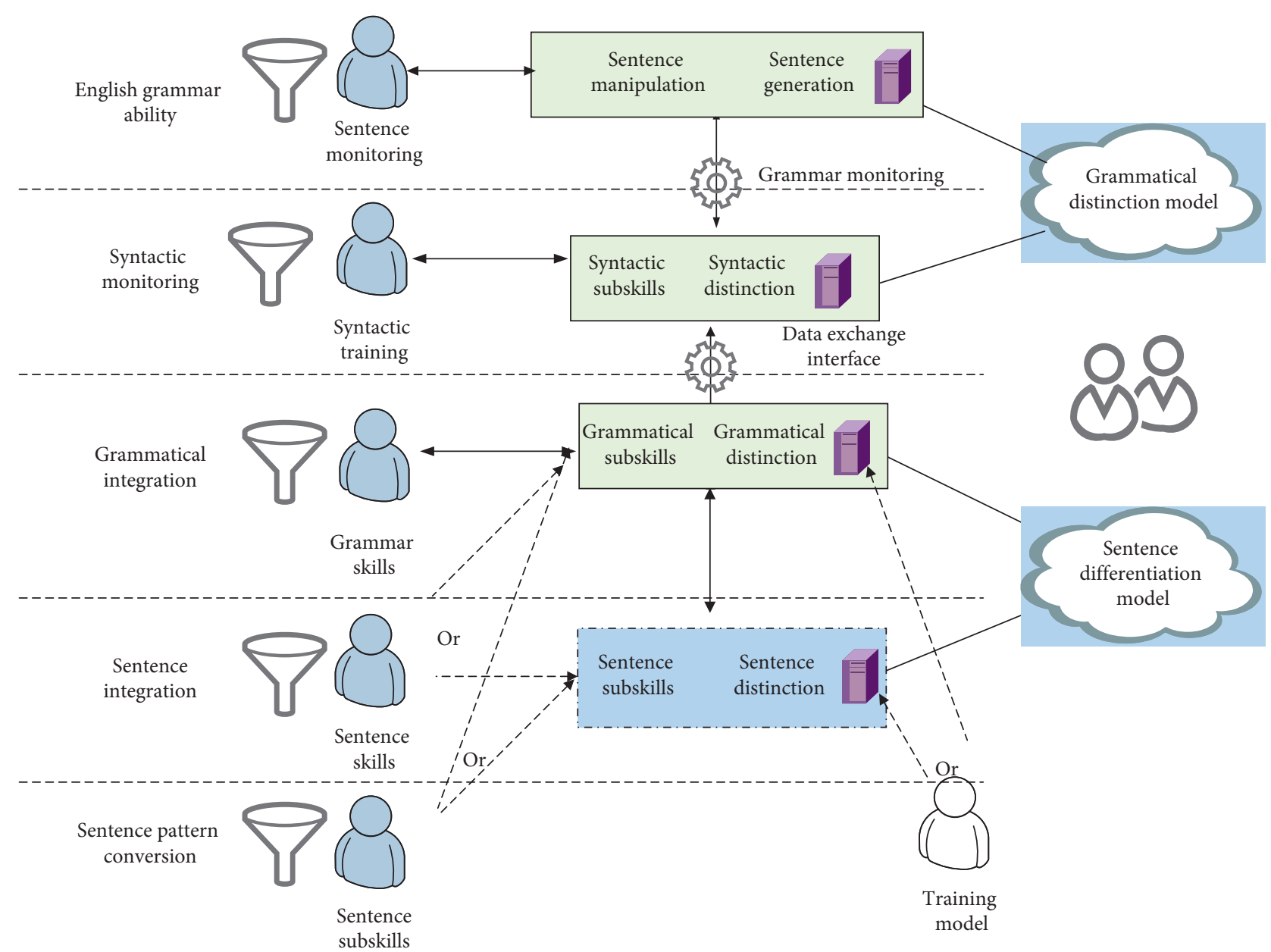

Figure 1: Network model of English grammar discriminating ability training, grammar skills, and grammar subskills discriminating training.

TABLe 1: General description of the design framework of the English Grammar discriminating ability training scale.

\begin{tabular}{|c|c|c|c|c|}
\hline $\begin{array}{l}\text { Measuring the } \\
\text { target }\end{array}$ & Measuring project & The test content & Test content description & Test type \\
\hline $\begin{array}{l}\text { Knowledge of } \\
\text { English grammar }\end{array}$ & $\begin{array}{l}\text { English sentence } \\
\text { monitoring skills }\end{array}$ & $\begin{array}{l}\text { English lexical } \\
\text { monitoring subskill } \\
\text { English syntax } \\
\text { monitoring subskill }\end{array}$ & $\begin{array}{c}\text { Using English lexical knowledge and rules } \\
\text { proficiently to identify and correct lexical errors } \\
\text { in sentences } \\
\text { Good command of English syntactic knowledge } \\
\text { and rules to identify and correct syntactic errors } \\
\text { in sentences }\end{array}$ & $\begin{array}{l}\text { Error identification } \\
\text { and correction }\end{array}$ \\
\hline \multirow[t]{2}{*}{$\begin{array}{l}\text { Use of English } \\
\text { grammar }\end{array}$} & $\begin{array}{l}\text { English sentence } \\
\text { operation skills }\end{array}$ & $\begin{array}{l}\text { English sentence } \\
\text { integration subskills } \\
\text { English sentence } \\
\text { pattern change } \\
\text { subskills }\end{array}$ & $\begin{array}{l}\text { Using English syntactic rules to combine simple } \\
\text { sentences with compound sentences } \\
\text { Skilled use of English syntactic rules to transform } \\
\text { clauses, phrases, or sentences with the same or } \\
\text { similar meaning but different syntactic structures }\end{array}$ & $\begin{array}{l}\text { Sentence to merge } \\
\text { Conversion of clauses, } \\
\text { phrases, or sentences }\end{array}$ \\
\hline & $\begin{array}{l}\text { English sentence } \\
\text { generation skills }\end{array}$ & $\begin{array}{l}\text { Skills of translating } \\
\text { Chinese into English }\end{array}$ & $\begin{array}{l}\text { Using English grammar knowledge and rules } \\
\text { proficiently to produce English sentences } \\
\text { according to English grammar }\end{array}$ & English translation \\
\hline
\end{tabular}

it is true, it can add mapping of (n, M) and the state $s$ changes. Otherwise, this mapping scheme is excluding (pruning). It is represented by the state of the search process, continuously selecting from the set of candidate matching points and trying not to add vertices in the old state to it, and using it in the process feasibility function to determine whether to add new vertices to implement changes to the vertices. This requires continuous recursion until the query graph traversal completes the algorithm flow, as shown in Algorithm 1. 


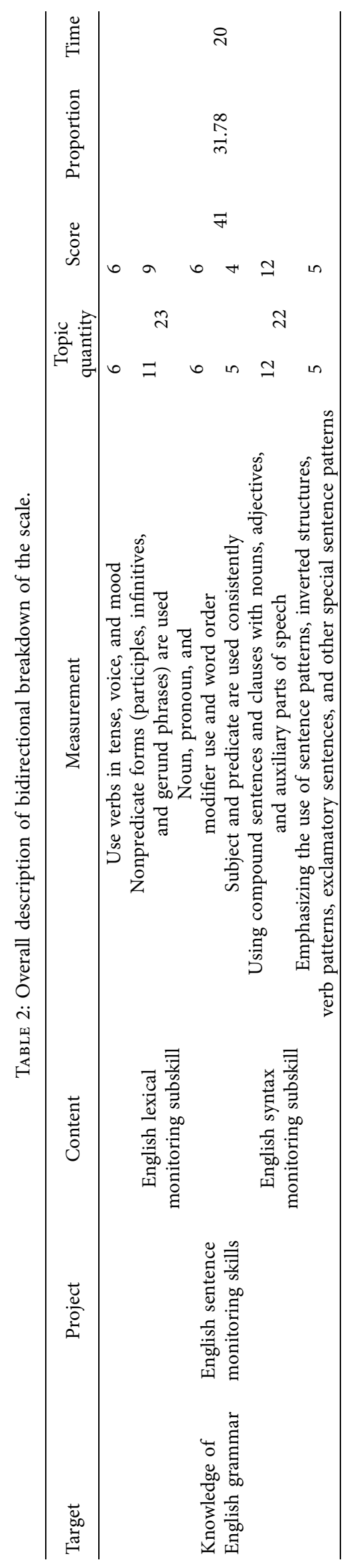


From the idea of VF2 algorithm, it can be seen that its core is actually search and pruning. After each compliant sentence of Uyghur nationality is analyzed by LTP neural network, the preprocessing result is obtained, and the preprocessing result is matched and converted with Chinese structure-type template rules. The automatic matching subprocess is a subgraph isomorphism decision-making problem. The template rule of the Chinese structure type in the transition graph is the query subgraph, and the preprocessing of the target tree is represented by the target graph; once the two graphs' isomorphism judgment says the match is successful, the transformation of Chinese structure template rule subprocesses can be continued. Otherwise, the preprocessing result is the dependency analysis result of the components in each clause of the final complex sentence.

\section{Search Filtering Based on English Grammar Discriminative Training}

The emotional color of language exists objectively. Therefore, English grammar discrimination training is usually not only a single description of events or characters but also conveys the standpoint, viewpoint, emotional attitude, and other information about the author himself or the group (faction) he represents. Finance, sports, culture, and entertainment are hot topics for people to search on the Internet, and related web pages are among the fastest growing categories. Relevant introductions, news events, social comments, etc. all have the author's emotional tendency to varying degrees. The media and the general public all need to timely understand these positive or negative reports and comments. The text is classified from the angle of orientation.

The main process of search filtering and classification of language grammar discrimination training is shown in Figure 3. As can be seen from the figure, the whole work is divided into two parts: a training process and classification process, which is roughly the same as the usual ATC technique. The difference is that a method of extracting tendentious feature is proposed. Because, in the actual web page, orientation feature information appears to be very few, some web pages do not even give feature information, and according to the automatic classification, the feature selection method is easy to be used as a routine to filter out irrelevant information, but these characteristics have a strong tendency to distinguish ability and, therefore, first of all, left alone to be extracted.

English grammar to distinguish between the training filtration process can be summarized as the English grammatical syntax analysis and semantic analysis to distinguish between training and fill in the corresponding framework and English grammar to distinguish the training feature extracting, calculate correlations for each slot, and then, calculate the training of relevant English grammar; finally, compared with the filter template, threshold filtering results are obtained. English grammar discrimination based on semantic analysis is divided into three steps: dividing the analysis unit, calculating the relevancy, and comparing the filtering threshold. The text is divided into several analysis units using characteristic vocabulary. Calculating correlation is divided into two stages, partial correlation and global relevance computing local relevancy computation phase on the analysis of the syntactic semantic analysis within the unit to extract the corresponding lattice, according to the mapping rules between semantic relations and slots, filling local semantic framework, and then, according to the matching distance function and correlation distribution, namely, calculating local semantic framework of relevance. Calculating global relevancy and the global semantic framework of the text is based on the collection of local relevancy of the analysis unit. In the last stage of comparison of filtering threshold, the relevancy between the threshold in the filtering template and the global semantic framework of the text is compared, and the English grammar discrimination training with successful matching is filtered.

The distance function is of the local frame fill. When a feature item vacancy occurs in the process of filling the frame according to the sentence, remote matching is needed, that is, a feature item in the same paragraph or even farther range that can reflect the corresponding tendency relationship is used to fill. The distance function is given as follows:

$$
F_{d i s}(S)=\left\{\begin{array}{l}
1, \text { Same } \\
0.5, \text { Before } \\
0.25, \text { Sentence } \\
0.1, \text { other }
\end{array} .\right.
$$

For different collections, different degrees of relevance are defined. The relevance function $R$ of each collection is defined as follows to reflect the importance of matching in the filling process:

$$
R(s)=\left\{\begin{array}{l}
\text { 2, Main, } \\
1, \text { Behavior }
\end{array}\right.
$$

The correlation RL of each local framework $\mathrm{F}$ is

$$
\mathrm{RL}=\frac{\sum R(s) F_{\mathrm{dis}}(S)}{\sum R(s)} \text {. }
$$

By calculating the relevancy of the local framework as mentioned above, the relevancy RG of the global semantic framework can be obtained by adding all the relevances:

$$
\mathrm{RG}=\sum \mathrm{RL}\left(F_{\text {dis }}(S)\right)
$$

The weight calculation formula of frame $\mathrm{F}$ is as follows:

$$
W=\frac{\sum \omega(t) F_{\mathrm{dis}}(S)}{\sum \omega(t)} .
$$

The weight of the calculated global context frame can effectively eliminate the misjudgment caused by citing arguments contrary to the overall tendency of English grammar discrimination training because the weight can offset the positive and negative to some extent.

\section{Example Verification}

In this experiment, 556 propaganda articles were selected from some web portals, among which 400 were used as training corpus, 609 were critical of Falun Gong, 420 were 


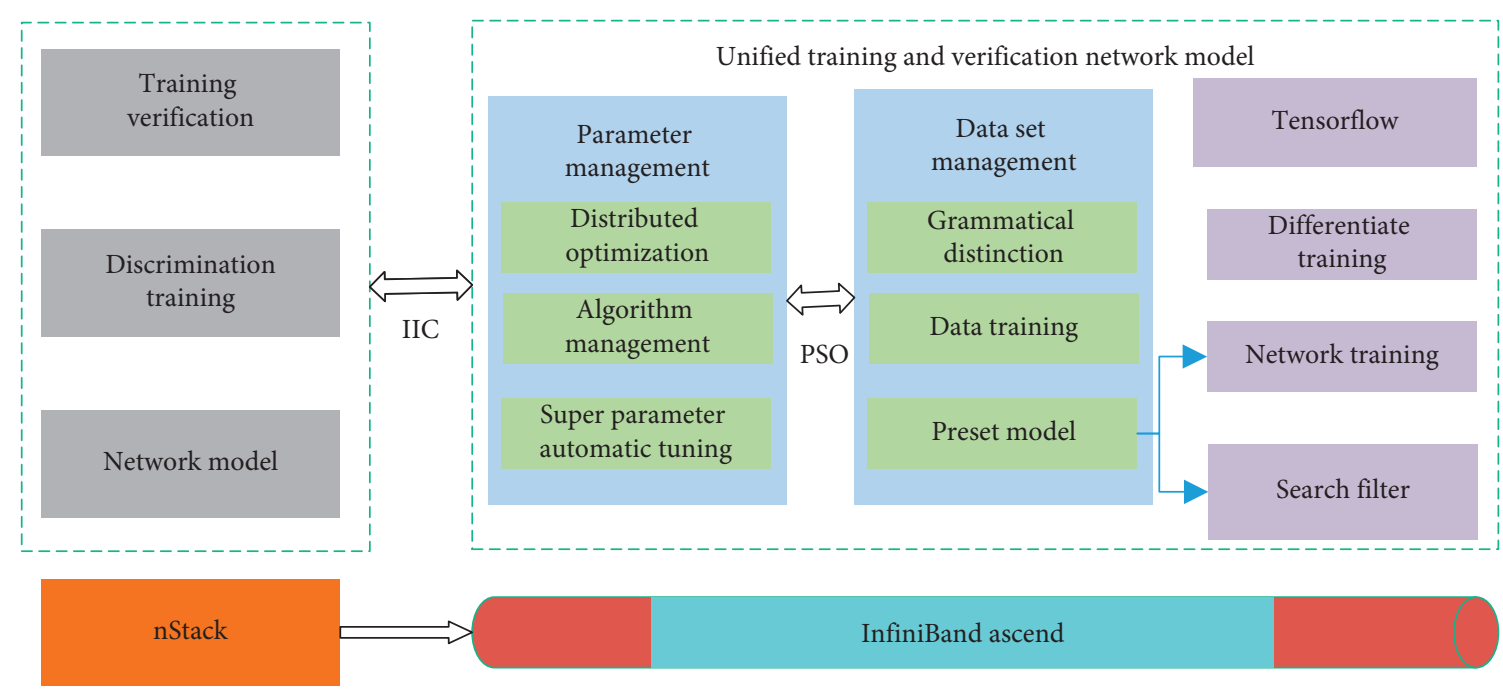

FIgURE 2: Network Model architecture diagram of English Grammar discriminative training.

used as training corpus, 198 were not related to Falun Gong, 100 were used as training corpus, and the rest were used as test corpus. The filtering system should release category 2 and 3 articles and reject category 1 articles to complete the filtering of undesirable information.

For the convenience of comparison, we compare the KNN classification method which achieves good results in theme-based information filtering with the semantic pattern analysis method adopted in this paper.

(1) The text $R$ to be filtered is entered.

(2) $\mathrm{R}$ is divided into analysis units.

(3) The frame of the analysis unit is matched, and the local relevancy is calculated.

(4) The global relevancy of the whole text is calculated.

(5) The relevancy is compared with the filtering threshold. If the filter meets the filtering criteria, the filter mark is given; otherwise, the pass mark is given.

In terms of evaluation indexes, we adopted two indexes, accuracy rate and recall rate, which were defined in this experiment as follows:

(1) Accuracy = number of successfully filtered articles/ number of labeled filtered articles

(2) Recall rate $=$ number of publicity articles successfully filtered/number of publicity articles in corpus

In order to enhance the precision of small-text filtering, we also make a distinction on whether the text length is considered in the final weight. In fact, whether the relevancy of the text to be filtered should consider the text length or not is also involved in the selection of the field value of the filter template.

In this way, for the text set to be filtered, the statistical $\mathrm{KNN}$ classification method is first used for experiments, and then, the semantic pattern analysis method adopted in this paper is used for experiments, from which the differences between the two in the biased text filtering are compared.
We divided the corpus used for testing into two groups; each group contains three different types of articles, the number of each type in the two groups is about the same, and the two groups of test sets are relatively independent; the purpose is to compare the difference between the statistical method under the same training set and the method based on semantic pattern analysis discussed in this paper. Figure 4 shows the experimental results on test set 1 , and Figure 5 shows the experimental results on test set 2.

From Figures 4 and 5, you can see that whatever the group test set is, the same training set is used in a certain area in this paper, the methods for better effect than the traditional statistical method, while statistical methods in the theme filter have achieved good effect, but its disadvantage exposed is it has a propensity to text filtering, without considering the semantic relationship between the features. However, the method based on semantic pattern analysis achieves an advantage in text filtering with strong tendency by accurately analyzing the semantic relationship between entities. Its accuracy is 9 percentage points higher than that of statistical methods on average, and it is also much faster than statistical methods in experiments. Of course, we need to do tests in different fields and on larger data sets to illustrate the problem more fully, but the method discussed in this paper has shown strong potential in the aspect of biased text filtering, which also lays a good foundation for further research.

In order to prevent the problem of overfitting in the process of data training, cross validation is adopted in the experiment. The dataset is divided into K groups, of which the 1 group is recycled as test data and the rest of the k-1 group is used as training data. In this way, $\mathrm{K}$ test results can be obtained, and the corresponding indexes of the system can be obtained by taking the average of them. In the experiment, by changing the proportion of the training dataset in the total experimental dataset, the influence of the training data amount on the recommended results was compared. 
(i) Input: Any intermediate state $\mathrm{Si}$; The initial state is $\mathrm{S} 0$

(ii) Output: Vertex mapping pairs for target graph G1 and query subgraph G2

(iii) $\operatorname{Match}(\mathrm{s})$

(iv) Begin

(v) If ( $1 \mathrm{VI}(\mathrm{S})$ contains all vertices in query subgraph G2)//G2 isomorphism in GI has been found

(vi) Output $M(\mathrm{~S}) ; / /$ Subgraph, end of search

(vii) Else

(viii) The matching point pair set $\mathrm{P}(\mathrm{S})$ is calculated according to the current local matching $\mathrm{M}(\mathrm{S})$.

(ix) For Each $p$ in $\mathrm{P}(\mathrm{S}) / /$ Traversal matching point pair set $\mathrm{F}(\mathrm{S})$

(x) If(in the case of $p=(\mathrm{n}, \mathrm{m}))$, the feasibility function $\mathrm{F}(\mathrm{S}, \mathrm{n}, \mathrm{m}\}=$ true $) / /$ If the matching $p$ is added, it is feasible

(xi) $\mathrm{s}^{\prime}=s \mathrm{U} p$; Match(s');//L adds $\mathrm{P}$ to $s$ and recursively calls Match(s) $\}$ to continue the search

(xii) End For Each

(xiii) Restore the data structure and trace back to the previous state.//No child of the isomorphism has been found after multiple calls to $\operatorname{Match}(\mathrm{s})$

(xiv) //Figure, which indicates that the current state cannot be expanded to be feasible

(xv) The knife graph isomorphism matches, then goes back to the previous state

(xvi) End

Algorithm 1: English grammar discriminative training network model algorithm.

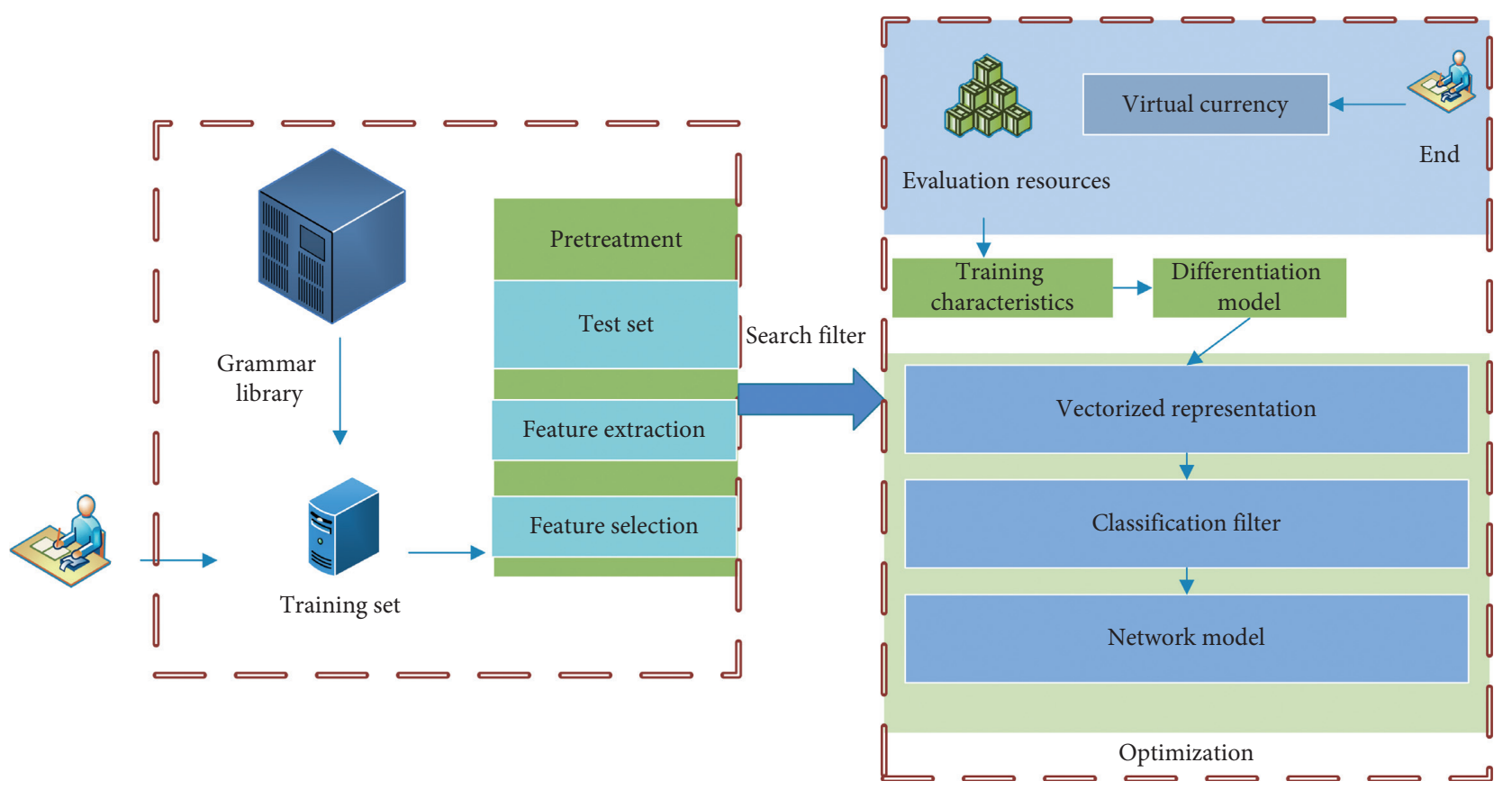

FIGURE 3: English Grammar discrimination training search filter.

Meanwhile, the precision and recall of the recommendation system are also related to the length of the returned tag list of the recommendation system. Therefore, in order to reflect the performance of the recommendation system more comprehensively, the influence of the length of different recommendation lists on the experimental results is also tested in the experiment. Figures 6 and 7 show the experimental results:

Through analysis of diagram 6, Figure 7 experimental results can be concluded as follows: search filtering system accuracy increased with the increase of return label number $n$, the recall rate increased with the increase of tag number $n$, the former is increased when recommending tag number $n$ will inevitably be some useless recommended results, and for the latter, apparently, when search filter tag number $n$ increases, search filter in the list of tags to cover users interested in grasping it will improve. In addition, as the number of test datasets of the search filtering system increases, the accuracy and recall rate of the search filtering system will also increase, proving that the performance of the search filtering system in this paper can be improved through continuous learning of historical data.

On the basis of the 500 grammatical distinctions of fuzzy retrieval given by 100 users, the description of users' personal characteristics and scenes is added and is entered into 


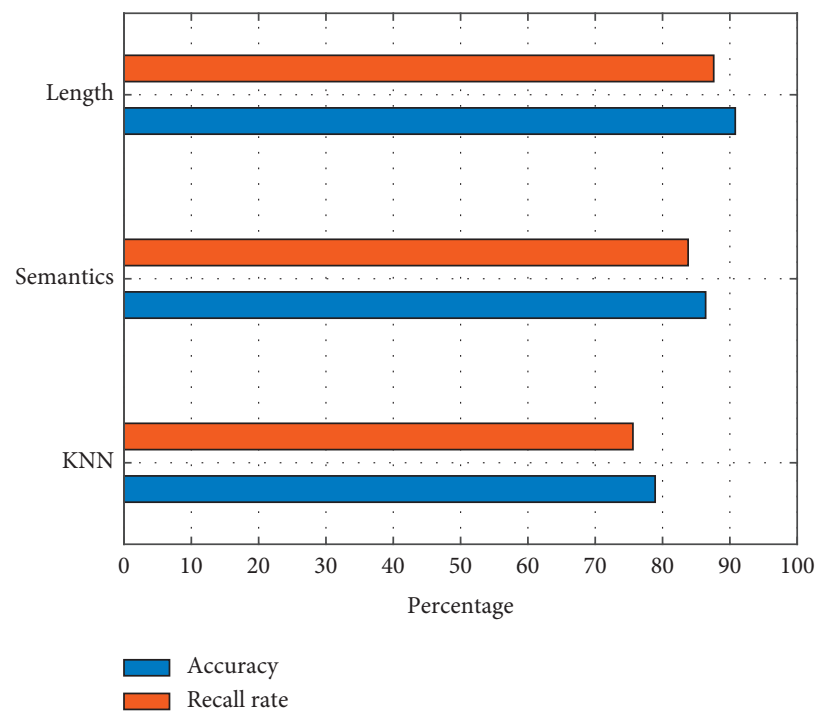

FIgURE 4: Search filtering experiment result 1.

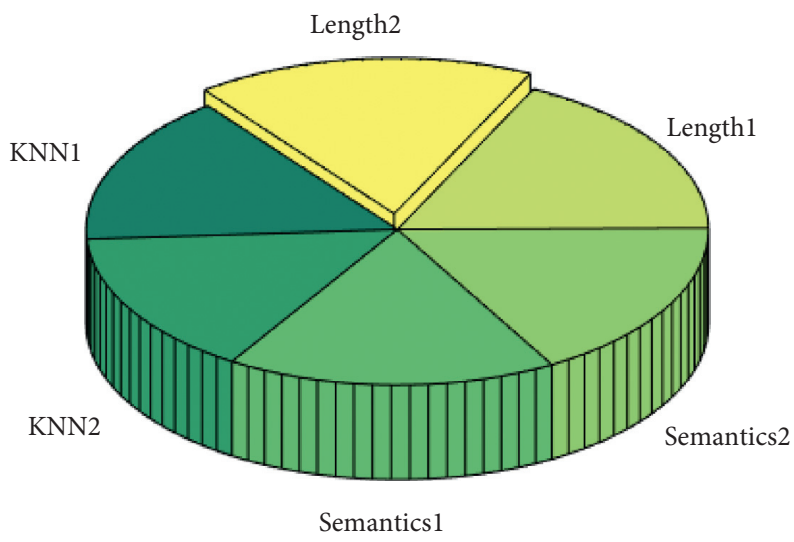

Figure 5: Results of the search filter experiment 2.

the search filter system as a keyword. By comparing the returned title of the search filter retrieval with the English grammar resources that the user is actually interested in, the retrieval system accuracy after adding the search filter function can be obtained, as shown in Figure 8.

According to the abovementioned experimental results, it can be seen that the retrieval accuracy of the search filtering system for fuzzy English grammar discrimination is slightly improved after docking with the recommendation system, indicating that the search filtering system has certain predictive sorting function for the resources that users are interested in on the basis of ensuring the original retrieval effect.

Finally, the accuracy of fuzzy text retrieval in the search and filter system with the function of synonym association can be obtained, as shown in Figure 9.

According to the analysis in Figure 9, it can be seen that the original Word2vec word vector direct correlation function is used to extend the semantic and grammatical discrimination and interest labels of search and screening.
As shown in the blue and red lines, the blue line represents the semantic and grammatical characteristics of expanded search and screening. It can be seen that it can improve the searching and filtering performance of the system, but it can also lead to a significant decrease in the precision of fuzzy searching and filtering. By using word2VEC directed association function, the accuracy of final search filtering results can be greatly improved. The reason is that the original word2vec lenovo results cannot guarantee that they correspond to the search key words in the filtration system resource, and even some irrelevant, ancient association results will have certain disturbance on the retrieval process, making some unrelated English grammar in the returned list with the user demand, resulting in a decline in search filtering accuracy. However, word2VEC's directional association function can make the associative words converge within the set range of keywords in the resource tag and semantically ensure that the words are close to the actual needs of users, so the search filtering resources obtained are more accurate. 


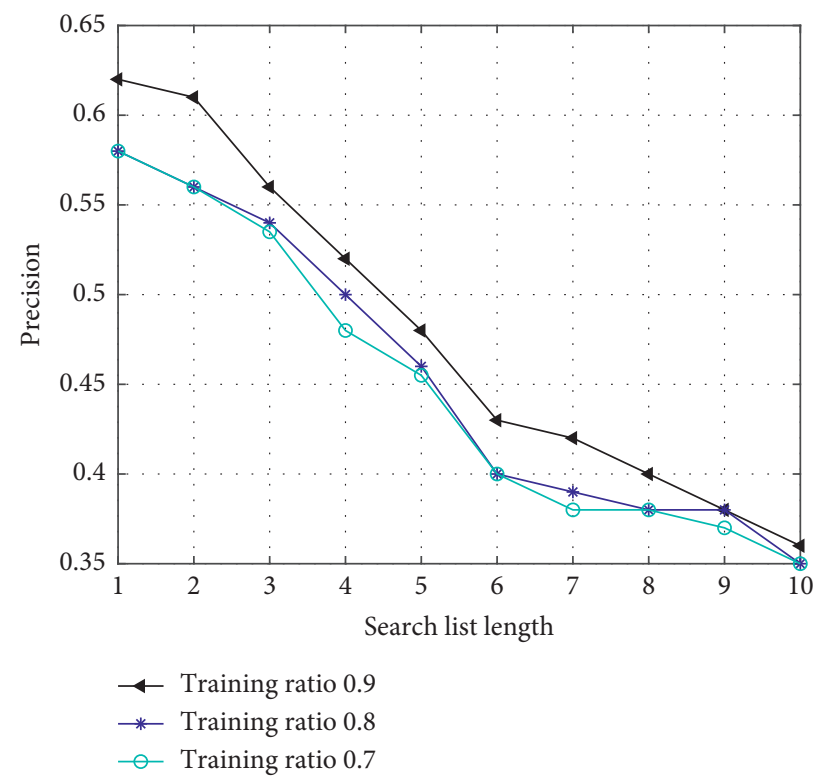

FIgURE 6: Search filtering accuracy of discriminative english grammar training.

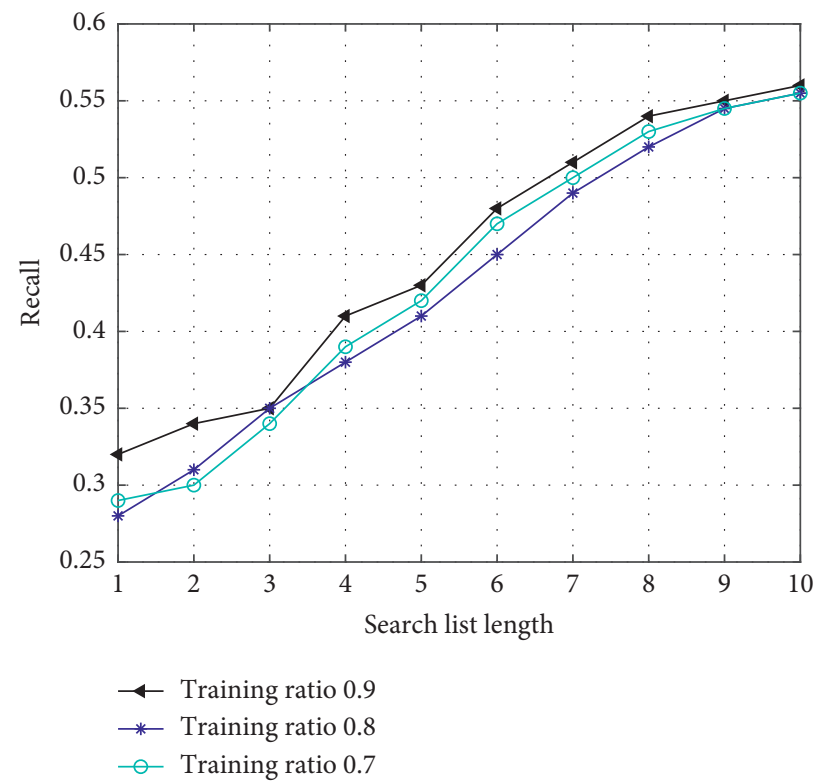

Figure 7: Recall rate of English grammar discriminative training search filter. 


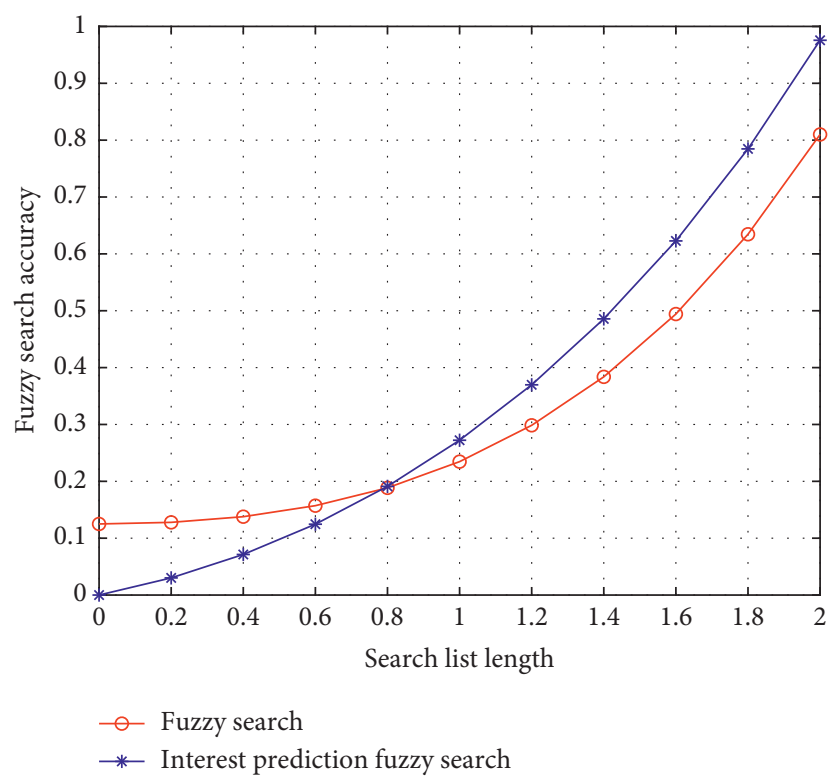

FIgURE 8: The discriminative search accuracy of fuzzy English grammar after interest prediction.

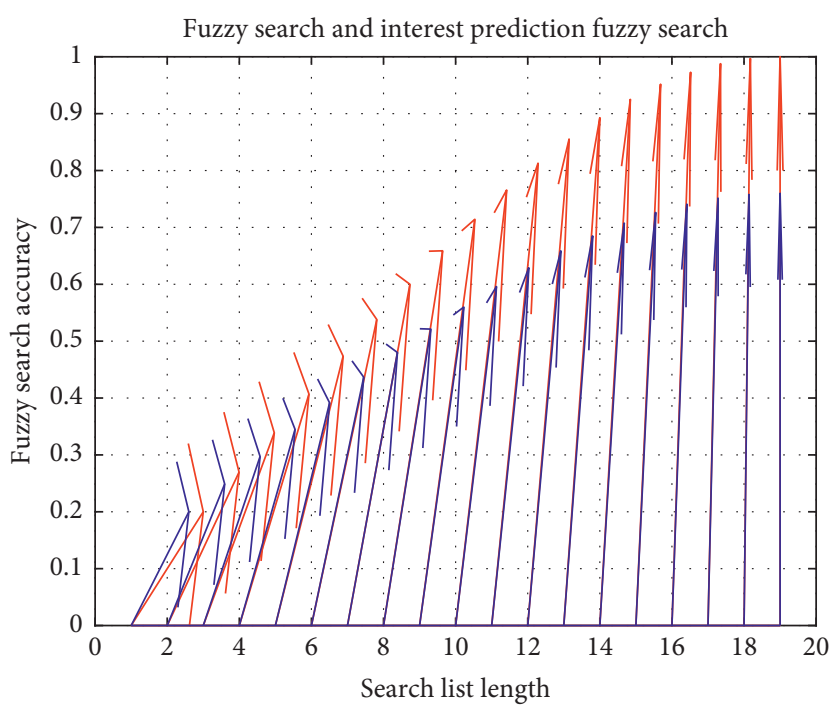

FIGURE 9: Fuzzy search accuracy after syntax extension.

\section{Conclusions}

Firstly, English grammar to distinguish the sexual training sentence analysis method theory knowledge is mainly discussed, and English grammar to distinguish the sexual training sentence analysis method of comprehensive analysis of the ideas about English grammar to distinguish sexual training important guiding significance to the Uighur dependent syntactic analysis is pointed out, and the structure of English grammar to distinguish sexual training type template is designed; secondly, in order to realize the rule conversion in the structure type template of English grammar discriminative training, a comprehensive analysis and postprocessing method which introduces the idea of VF2 algorithm is proposed. Then, combining the postprocessing algorithm of comprehensive analysis with the single sentence dependency analysis model of neural network, a complex sentence dependency analysis method combining the postprocessing of comprehensive analysis with the neural network model is proposed. Finally, the validity and feasibility of this method are verified by a large number of corpus experiments. Finally, the discriminative training model of Word2VEC English grammar is studied in depth, and the function of directed association of word vectors is proposed innovatively according to the actual needs, which is combined with the search filter system. While improving the accuracy of system search, it also reduces the computing time, resources were required by system synonym search, and it improves the overall efficiency of system operation. In the aspect of machine learning, template learning is closely related to text representation, and how to learn through user feedback 
is still a topic worthy of attention. In addition, since users' interests are not invariable in the filtering process, this is also the content of the next research.

\section{Data Availability}

The data used to support the findings of this study are available from the corresponding author upon request.

\section{Conflicts of Interest}

The author declares that there are no known conflicts of interest.

\section{Acknowledgments}

This work was supported by the Research project of $\mathrm{Hu}$ manities and Social Sciences in Colleges and Universities in Henan Province: Research on Writing Strategies in online English Writing Model from the Perspective of Activity Theory (2021-ZZJH-209).

\section{References}

[1] W. Guoqing, "Changming. Learning a compact vein discrimination model with GANerated samples," IEEE Transactions On Information Forensics and Security, vol. 15, pp. 635-650, 2019.

[2] T.-K. Eom, "Understanding-based discovery learning in English grammarbooks," Journal of Education \& Culture, vol. 21, no. 6, pp. 321-348, 2015.

[3] I. Amour and T. Kauranne, "A variational ensemble Kalman filtering method for data assimilation using $2 \mathrm{D}$ and $3 \mathrm{D}$ version of COHERENS model," International Journal for Numerical Methods in Fluids, vol. 83, no. 6, pp. 544-558, 2017.

[4] D. Pohl, A. Bouchachia, and H. Hellwagner, "Online indexing and clustering of social media data for emergency management," Neurocomputing, vol. 172, no. 8, pp. 168-179, 2016.

[5] P. Thomas and M.-C. Suhner, "A new multilayer perceptron pruning algorithm for classification and regression applications," Neural Processing Letters, vol. 42, no. 2, pp. 437-458, 2015.

[6] D. Cárdenas-Peña, D. Collazos-Huertas, A. Álvarez-Meza, and G. Castellanos-Dominguez, "Supervised kernel approach for automated learning using General Stochastic Networks," Engineering Applications of Artificial Intelligence, vol. 68, pp. 10-17, 2018.

[7] D. T. S. Kumar, "Video based traffic forecasting using convolution neural network model and transfer learning techniques," Journal of Innovative Image Processing, vol. 2, no. 3, pp. 128-134, 2020.

[8] B. Han, X. Gao, and X. Cui, "Model-based sensitivity analysis on aerosol optical thickness prediction," International Journal of Distributed Sensor Networks, vol. 2015, no. 1, pp. 31-39, 2015.

[9] Y. Wu, H. Wang, and B. Zhang, "Using radial basis function networks for function approximation and classification," Isrn Applied Mathematics, vol. 2012, pp. 1089-1122, 2015.

[10] F. Santoso and S. J. Redmond, "Indoor location-aware medical systems for smart homecare and telehealth monitoring: state-of-the-art," Physiological Measurement, vol. 36, no. 10, pp. 53-68, 2015.
[11] Y. Chen, L. Zhu, P. Ghamisi, X. Jia, G. Li, and L. Tang, "Hyperspectral images classification with gabor filtering and convolutional neural network," IEEE Geoscience and Remote Sensing Letters, vol. 14, no. 12, pp. 2355-2359, 2017.

[12] G. Langrock, N. Wiehl, H.-O. Kling et al., "Digital liquidscintillation counting and effective pulse-shape discrimination with artificial neural networks," Radiochimica Acta, vol. 103, no. 1, pp. 15-25, 2015.

[13] G. M. Stanley, "Big Data Approximating Control (BDAC) - a new model-free estimation and control paradigm based on pattern matching and approximation," Journal of Process Control, vol. 67, pp. 23-42, 2017.

[14] A. Arkoma, M. Hänninen, K. Rantamäki, J. Kurki, and A. Hämäläinen, "Statistical analysis of fuel failures in large break loss-of-coolant accident (LBLOCA) in EPR type nuclear power plant," Nuclear Engineering and Design, vol. 285, pp. 1-14, 2015.

[15] H. Xiao, B. Biggio, B. Nelson, H. Xiao, C. Eckert, and F. Roli, "Support vector machines under adversarial label contamination," Neurocomputing, vol. 160, pp. 53-62, 2015.

[16] I. Ghalehkhondabi, E. Ardjmand, and G. R. Weckman, "An overview of energy demand forecasting methods published in 2005-2015," Energy Systems, vol. 8, no. 2, pp. 31-67, 2016.

[17] J. Zhao, J. Wang, and F. Liu, "Multistep forecasting for shortterm wind speed using an optimized extreme learning machine network with decomposition-based signal filtering," Journal of Energy Engineering, vol. 142, no. 3, pp. 40150361401503621, 2016.

[18] N. Moritz, B. Kollmeier, and J. Anemuller, "Integration of optimized modulation filter sets into deep neural networks for automatic speech recognition," IEEE/ACM Transactions on Audio, Speech, and Language Processing, vol. 24, no. 12, pp. 2439-2452, 2016.

[19] F. M. Santin, R. V. Da Silva, and J. M. V. Grzybowski, "Artificial neural network ensembles and the design of performance-oriented riparian buffer strips for the filtering of nitrogen in agricultural catchments," Ecological Engineering, vol. 94, pp. 493-502, 2016.

[20] S. Lee, J. Cha, M. K. Kim, K. S. Kim, V. H. Pham, and M. Leach, "Neural-network-based building energy consumption prediction with training data generation," Processes, vol. 7, no. 10, pp. 731-748, 2019.

[21] S. S. Rautaray and A. Agrawal, "Vision based hand gesture recognition for human computer interaction: a survey," Artificial Intelligence Review, vol. 43, no. 1, pp. 61-114, 2015.

[22] F. Li, H. Bai, and Y. Zhao, "FilterNet: adaptive information filtering network for accurate and fast image super-resolution," IEEE Transactions on Circuits and Systems for Video Technology, vol. 30, no. 6, pp. 1511-1523, 2020.

[23] D. Chagas-Paula, T. Oliveira, T. Zhang, R. Edrada-Ebel, and F. Da Costa, "Prediction of anti-inflammatory plants and discovery of their biomarkers by machine learning algorithms and metabolomic studies," Planta Medica, vol. 81, no. 06, pp. 450-458, 2015.

[24] X. Li, X. Cheng, and S. Su, "A hybrid collaborative filtering model for social influence prediction in event-based social networks," Neurocomputing, vol. 230, pp. 197-209, 2016.

[25] Y. Zhang, T. Shen, X. Ji, Y. Zhang, R. Xiong, and Q. Dai, "Residual highway convolutional neural networks for in-loop filtering in HEVC," IEEE Transactions on Image Processing, vol. 27, no. 8, pp. 3827-3841, 2018.

[26] B. Song, Y. Gao, and X.-M. Li, "Research on collaborative filtering recommendation algorithm based on mahout and 
user model," Journal of Physics: Conference Series, vol. 1437, no. 1, pp. 012095-012101, 2020.

[27] X. U. Xing, "Study on discrimination model and application of mine water inrush source based on swarm intelligent neural network," Acta Geologica Sinica(English Edition), vol. 93, no. z2, pp. 635-650, 2019. 